\title{
Patient Complexity: More Than Comorbidity. The Vector Model of Complexity
}

\author{
Monika M. Safford, $M D^{1,2}$, Jeroan J. Allison, MD, MSc ${ }^{2}$, and Catarina I. Kiefe, $P h D, M D^{1,2}$ \\ 'Deep South Center on Effectiveness at the Birmingham VA Medical Center, Birmingham, AL, USA; ${ }^{2}$ University of Alabama at Birmingham, \\ Birmingham, AL, USA.
}

BACKGROUND: The conceptualization of patient complexity is just beginning in clinical medicine.

OBJECTIVES: This study aims (1) to propose a conceptual approach to complex patients; (2) to demonstrate how this approach promotes achieving congruence between patient and provider, a critical step in the development of maximally effective treatment plans; and (3) to examine availability of evidence to guide trade-off decisions and assess healthcare quality for complex patients.

METHODS/RESULTS: The Vector Model of Complexity portrays interactions between biological, socioeconomic, cultural, environmental and behavioral forces as health determinants. These forces are not easily discerned but exert profound influences on processes and outcomes of care for chronic medical conditions. Achieving congruence between patient, physician, and healthcare system is essential for effective, patientcentered care; requires assessment of all axes of the Vector Model; and, frequently, requires trade-off decisions to develop a tailored treatment plan. Most evidence-based guidelines rarely provide guidance for trade-off decisions. Quality measures often exclude complex patients and are not designed explicitly to assess their overall healthcare.

CONCLUSIONS/RECOMMENDATIONS: We urgently need to expand the evidence base to inform the care of complex patients of all kinds, especially for the clinical trade-off decisions that are central to tailoring care. We offer long- and short-term strategies to begin to incorporate complexity into quality measurement and performance profiling, guided by the Vector Model. Interdisciplinary research should lay the foundation for a deeper understanding of the multiple sources of patient complexity and their interactions, and how provision of healthcare should be harmonized with complexity to optimize health.

KEY WORDS: patient complexity; evidence-based care;

Vector Model of Complexity.

J Gen Intern Med 22(Suppl 3):382-90

DOI: $10.1007 /$ s1 1606-007-0307-0

(C) Society of General Internal Medicine 2007

\section{INTRODUCTION}

Complex patients are increasingly common in clinical medicine. Patients made complex by multiple cooccuring medical conditions have been the focus of recent discussions, with concerns expressed about potential vulnerability created by the current approach to quality of care assessment and accountability in health care. ${ }^{1-4}$ However, socioeconomic, cultural, behavioral, and environmental circumstances also contribute to complexity, but these latter sources of complexity and their ramifications have not received similar attention. In fact, there is currently no widely accepted conceptualization that portrays the numerous influences that together make a patient more or less complex.

Patient complexity from any source may affect process or outcomes of care, resulting in the need to adjust care plans. Clinical guidelines have become essential tools for providing evidence-based care, but few provide guidance for making trade-off decisions, either to individual providers or to health systems. ${ }^{1,2}$ Furthermore, current quality measures do not incorporate complexity, ${ }^{3}$ and the recent trend toward tying reimbursement to disease-specific quality measures may increase attention to improving the measures at the possible expense of patient-centered, individually tailored care. ${ }^{2-4}$

In this paper, we propose the Vector Model of Complexity, a conceptual model that defines complexity along axes representing major determinants of health. We demonstrate that the patient's complexity along numerous axes has profound implications for the delivery of effective chronic health care. We discuss how the Vector Model can be used to maximize the impact of medical recommendations on health outcomes. Then, we examine how well evidence-based tools help clinicians and health systems to accomplish this. Finally, we propose strategies to integrate the Vector Model into the process and assessment of healthcare delivery to maximize the quality of chronic care for complex patients, a large and growing number of Americans. ${ }^{5}$

\section{CONCEPTUAL FRAMEWORK OF PATIENT COMPLEXITY}

The American Heritage Dictionary defines "complex" as "consisting of interconnected or interwoven parts; involved or intricate, as in structure; complicated." While all humans are inherently complex, some patients are more challenging to care for than others. What then makes some more complex than others? 
In answer, consider first the determinants of health, which include biology/genetics, socioeconomics, culture, environment/ ecology, behavior, and the medical system. ${ }^{6-10}$ A common model is depicted in Figure 1.

The associations of each determinant with health outcomes have been examined at the population level. ${ }^{7,11,12}$ However, the contribution from each determinant may vary in individual patients, and the relationships between the domains of determinants is not provided by the schematic diagram. The overall effect of the various determinants is therefore unclear. Furthermore, the model does not demonstrate how clinicians or the healthcare system should modify their interactions with patients, given the mix of determinants present, to optimize the health of the patient.

To address these needs, we propose the Vector Model of Complexity (Fig. 2), using the principles of vector physics. Each health determinant from Figure 1 (except healthcare) is conceptualized as exerting its influence along an axis, which intersects with the other determinants' axes at a central point, the patient. Complexity can exist along each axis as a force which has both magnitude and directionality, defining vector $\mathbf{v}$. At any given time, a particular vector may exert a force increasing complexity, or alternatively, lessening complexity. In vector physics, an overall vector can be summed together (see also Appendix); in the Vector Model, we conceptualize overall complexity as being similarly determined by multiple components. While the summary vector in physics can be precisely calculated, we do not know the mathematical relationships between the vectors of patient complexity. Nevertheless, the concept of a formal relationship among the different domains of complexity is clinically useful: according to the Vector Model of Complexity, two patients with similar biologic complexity may present greater or less overall complexity along any given axis, at a given encounter, depending on the relative sum of vectors at that time (Fig. 2).

The Vector Model provides a basis from which to examine how the sixth determinant of health, medical care, should be adapted to maximize its impact on health. Clinicians focus primarily on the biological axis consistent with the emphasis in allopathic medical education on the biology of disease; however, influences along the other axes can either augment or diminish complexity along the biological axis. This results in similar treatment plans producing different results in patients with differing complexity. Assessment of all the axes of complexity may be an essential step in developing effective treatment plans for individual patients.

The interrelatedness between the axes is a key feature of the Vector Model, distinguishing it from previous conceptualizations of the determinants of health. The Vector Model explicitly acknowledges that the determinants are related. For example, recent immigrants may live in impoverished, environmentally

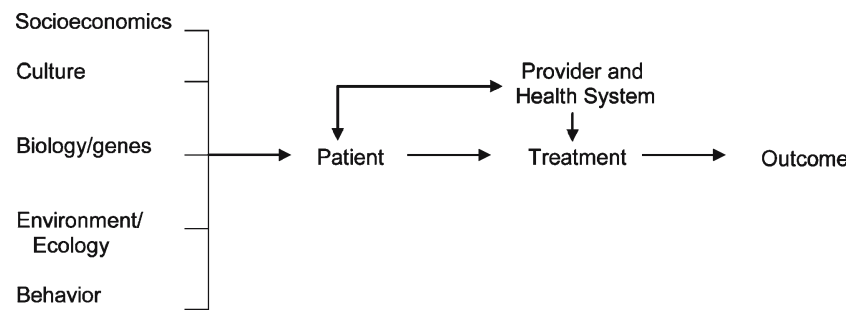

Figure 1. Outcomes oriented model of the determinants of health disadvantageous locations (such as inner city slums); to have restricted financial means; and to lack health insurance. Cultural influences may exert pressures toward tobacco use, unhealthy diets, and mistrust in an unfamiliar approach to medical care. Cultural complexity therefore influences socioeconomic, environmental, and behavioral complexity. To represent these relationships, we add a web to the intersecting axes. The web has several appealing relevant features. Although they are often difficult to discern, the strands connect every aspect of the web, with each axis linked directly with all others, so that when one part of the web is perturbed, the entire web may be affected. The interconnections superficially appear tenuous, but may in fact be quite tenacious (Fig. 2).

Table 1, organized by complexity vector, lists examples of factors that contribute to complexity, leading to differential effectiveness of medical treatment. Importantly, the Vector Model allows an individual's level of complexity to vary over time, reflecting the dynamic nature of complexity. For example, individuals newly diagnosed with diabetes must make considerable efforts to modify diet and exercise, and to monitor blood sugar. Once incorporated into the patient's lifestyle, these salutary health behaviors tend to diminish complexity at future encounters. Each encounter with the healthcare system can be thought of as a series of vector diagrams. Whether the provider and healthcare system prove helpful or effective depends upon both (1) the complete assessment of the patient's complexity, and (2) the provider and healthcare system being equipped to respond.

\section{The Vector Model in Action: Illustrative Case Vignettes}

To better illustrate how these influences interact in patients, consider two illustrative cases (Fig. 3).

Mr. Smith has biological complexity, with multiple newly recognized conditions. However, his personal, family, and community circumstances exert stabilizing and supportive forces along social, cultural and environmental axes, diminishing the overall complexity confronting his treating physicians. Mr. Smith has the means to find culturally concordant doctors whom he can trust, enhancing motivation to quit smoking, make lifestyle changes, and take his medicines. His overall complexity vector along the biological axis is low (Fig. 4), and his treating physicians need not modify their usual approach.

In contrast, Mr. Jones has similar biological forces, but his other axes contribute forces toward greater complexity. Mr. Jones' social support is much less than Mr. Smith's. He has limited finances and may have difficulty paying for all the recommended medications, new diet, and follow-up care. He must balance his own self-care needs with his wife's. His environment makes it difficult to exercise, and an unsafe neighborhood may contribute to stress. Mr. Jones' overall complexity is greater than Mr. Smith's.

Without a very different approach by his treating physicians than that used for Mr. Smith, Mr. Jones may conclude that his doctors do not understand his situation, become overwhelmed, and lose motivation to quit smoking and make difficult lifestyle changes. The Vector Model of Complexity therefore explains why a disease-focused approach (that is, considering the biological vector alone) works well in Mr. Smith but fails in Mr. Jones. 


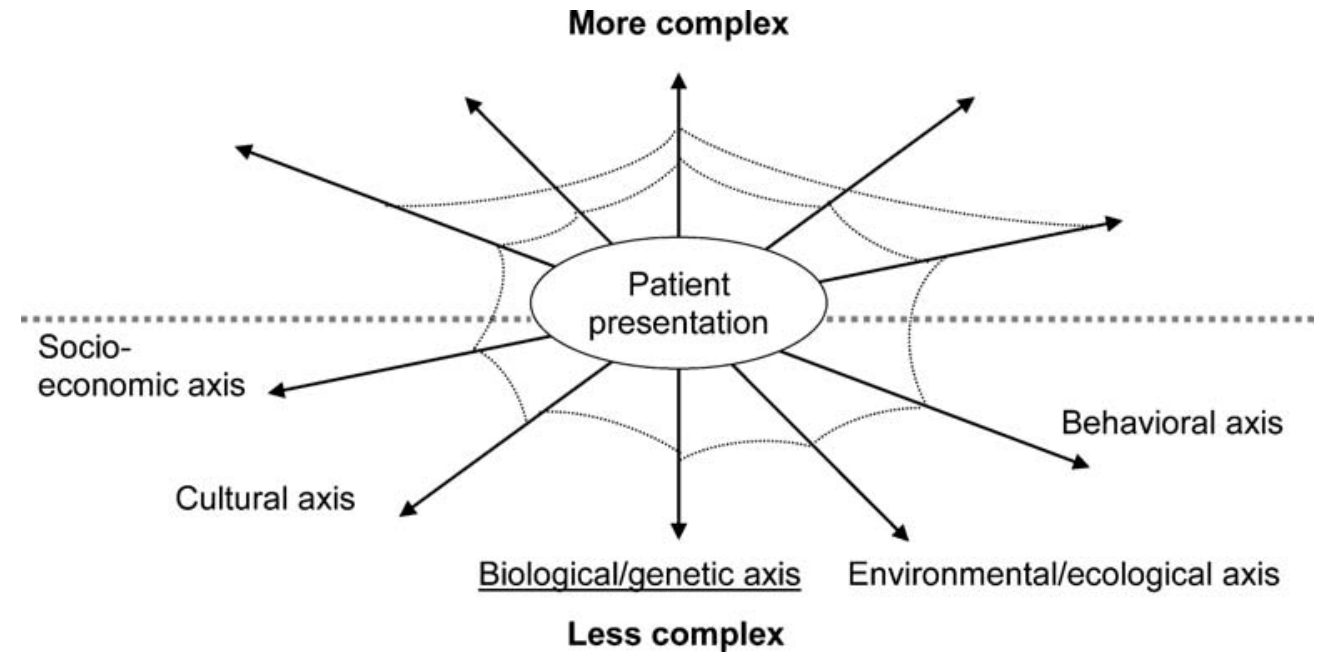

Figure 2. Interrelationships between influences on health that can lead to a complex patient: the Vector Model of Complexity. A patient's complexity arises from different axes, but physicians often focus on the biological axis (underlined). Each axis is related directly to the others, represented by the strands of a web that link both neighboring axes and axes that are not depicted as direct neighbors. For simplicity, we have shown only the strands between the biological axis and each of the others; in fact, we envision such strands to tie each axis to every other. Both the angles between each axis and the others, and therefore their relative situation to the other axes, and the tenacity of the strands tying the axes could be estimated through empiric work

\section{Using the Vector Model to Achieve Congruence}

Explanatory models of sickness, a medical anthropological concept, ${ }^{13}$ provide a mechanism to use the Vector Model to create effective treatment plans. According to these explanatory models, "physicians diagnose and treat diseases (abnormal-

Table 1. Examples of Contributors to Differential Medical Treatment Effects and of their Consequences, by Patient Complexity Component Vector

\begin{tabular}{|c|c|c|}
\hline Vector & $\begin{array}{l}\text { Sources of increased } \\
\text { complexity along the } \\
\text { Vector }\end{array}$ & $\begin{array}{l}\text { Specific } \\
\text { consequence }\end{array}$ \\
\hline \multirow[t]{3}{*}{ Socioeconomics } & $\begin{array}{l}\text { Lack of health } \\
\text { insurance }\end{array}$ & $\begin{array}{l}\text { Difficulty affording } \\
\text { treatment }\end{array}$ \\
\hline & Lack of transportation & $\begin{array}{l}\text { Difficulty accessing } \\
\text { providers }\end{array}$ \\
\hline & $\begin{array}{l}\text { Low educational } \\
\text { attainment }\end{array}$ & $\begin{array}{l}\text { Inability to navigate } \\
\text { complex systems }\end{array}$ \\
\hline \multirow[t]{3}{*}{ Culture } & Race/ethnicity & $\begin{array}{l}\text { Care that is not } \\
\text { culturally sensitive }\end{array}$ \\
\hline & Language & $\begin{array}{l}\text { Communication } \\
\text { barriers }\end{array}$ \\
\hline & Communication & $\begin{array}{l}\text { Distrust, perceived } \\
\text { discrimination }\end{array}$ \\
\hline \multirow[t]{3}{*}{$\begin{array}{l}\text { Biology/ } \\
\text { genetics }\end{array}$} & Multiple comorbidities & $\begin{array}{l}\text { Medication } \\
\text { interactions }\end{array}$ \\
\hline & Genetic variability & $\begin{array}{l}\text { Cannot achieve } \\
\text { recommended targets }\end{array}$ \\
\hline & Cognitive impairment & $\begin{array}{l}\text { Inability to follow } \\
\text { recommendations }\end{array}$ \\
\hline \multirow{3}{*}{$\begin{array}{l}\text { Environment/ } \\
\text { ecology }\end{array}$} & Pollution & Exposure to toxins \\
\hline & Neighborhood violence & Inability to exercise \\
\hline & $\begin{array}{l}\text { Lack of public } \\
\text { transportation }\end{array}$ & $\begin{array}{l}\text { Inability to buy } \\
\text { healthy foods }\end{array}$ \\
\hline \multirow[t]{3}{*}{ Behavior } & Smoking tobacco & $\begin{array}{l}\text { Cardiovascular, } \\
\text { pulmonary disease }\end{array}$ \\
\hline & Unhealthy diet & Obesity \\
\hline & Lack of physical activity & Diabetes \\
\hline
\end{tabular}

ities in the structure and function of body organs and systems), whereas patients suffer illnesses (experiences of disvalued changes in states of being and in social function)". ${ }^{14}$ Explanatory models of sickness fit well into the Vector Model of Complexity: they are determined by culture (national, racial, ethnic, occupational, or professional), religious beliefs, education and knowledge, social class [socioeconomic and cultural vectors]; gender, age, and personality traits [biological and behavioral vectors].

Hence, an important goal of the medical encounter is for the doctor and patient to develop "congruence," or, a shared view of realistically attainable health care goals. The physician must assess each axis of the Vector Model, harmonizing the treatment plan accordingly. Congruence may enhance trust, satisfaction, respect for preferences, adherence with recommendations, salutary self-management behaviors, and mutuality in the evaluation of treatment outcomes. ${ }^{12-17}$ The influence of trust and perceived discrimination on adherence with treatment plans and with health outcomes supports the importance of congruence. ${ }^{11}$

The healthcare system must also be designed to promote congruence. While some healthcare systems can provide successful care to individuals with, for example, addiction, homelessness, and culturally unique needs, others can be distinctly unaccommodating for such patients.

Failure to achieve congruence between patient and provider may provide insight into why half of patients with chronic disease do not take medications as directed. ${ }^{18}$ Noncongruence may contribute to differential use of preventive services, diagnostic procedures, and therapeutic interventions by race/ethnicity, even when access to care, diagnosis, and severity of illness are similar. In fact, patient "non-adherence," may be an excellent signal of (1) incomplete assessment of complexity and failure to modify the clinical approach accordingly, and/or (2) a healthcare system that remains ill-tooled to address the needs of complex patients. 
Case 1.

Mr. Smith is a 56 year old African American man recently discharged from the hospital after suffering a heart attack. He was given medications to control his blood pressure and lipids, and advised to take aspirin, as well as to quit smoking. While in the hospital, he was also diagnosed with diabetes, and given much information about changing his diet and exercising. He lives in a suburban neighborhood and has a stable employment situation with good health insurance benefits. He has a supportive wife and family.

Case 2.

Mr. Jones is a 56 year old European American man recently discharged from the hospital after suffering a heart attack. He was given medications to control his blood pressure and lipids, and advised to take aspirin, as well as to quit smoking. While in the hospital, he was also diagnosed with diabetes, and given much information about changing his diet and exercising. He lives in an inner city impoverished neighborhood with a sick wife who requires much care. He works as a truck driver, and has limited health insurance benefits.

Figure 3. Two patients with similar biologic but dissimilar socioeconomic, culture, environmental, and behavioral complexity

Unfortunately, physicians receive little training on how to achieve congruence. While cultural competency is increasingly being added to educational curricula, these programs have unknown success. ${ }^{19}$ In fact, it is not clear how each axis of the Vector Model should be assessed, and validated instruments to capture aspects of each axis are primarily designed for research purposes. Evidence-based guidelines are a cornerstone to guide clinical management; how well do they help physicians care for complex patients?

\section{GUIDANCE FOR IMPLEMENTING EVIDENCE-BASED CARE FOR COMPLEX PATIENTS}

Complex patients do benefit from evidence-based care. Although relatively few in number, clinical trials conducted in real-life settings demonstrated important clinical benefits ${ }^{20-25}$; a good example is the antihypertensive and lipid lowering to prevent heart attack trial (ALLHAT). Effective interventions to help individuals modify health-related behavior including physical activity, diet, and smoking exist. ${ }^{26,27}$ Likewise, trials conducted in socioeconomically and culturally complex patients suggest benefits, if individuals can be sufficiently engaged to become active, motivated participants. ${ }^{28-33}$ Even individuals with complex environmental exposures, such as inner city asthma patients, benefit from evidence-based care. ${ }^{29,30}$

Moreover, systems can be redesigned specifically to assure that key healthcare processes are accomplished in complex patients. ${ }^{34}$ Specific system interventions with promising results include colocation of services for persons with combined substance abuse and medical illness ${ }^{35,36}$; medical respite care as an adjunct to primary medical care for homeless persons ${ }^{37}$; and group visits for disadvantaged diabetic individuals. ${ }^{38}$

While evidence-based care works in complex patients and system redesign is possible, important gaps remain. The tradeoff decisions central to tailoring treatment plans should be informed by answering questions such as: Does time to achieve benefits differ across types of patients? Are some

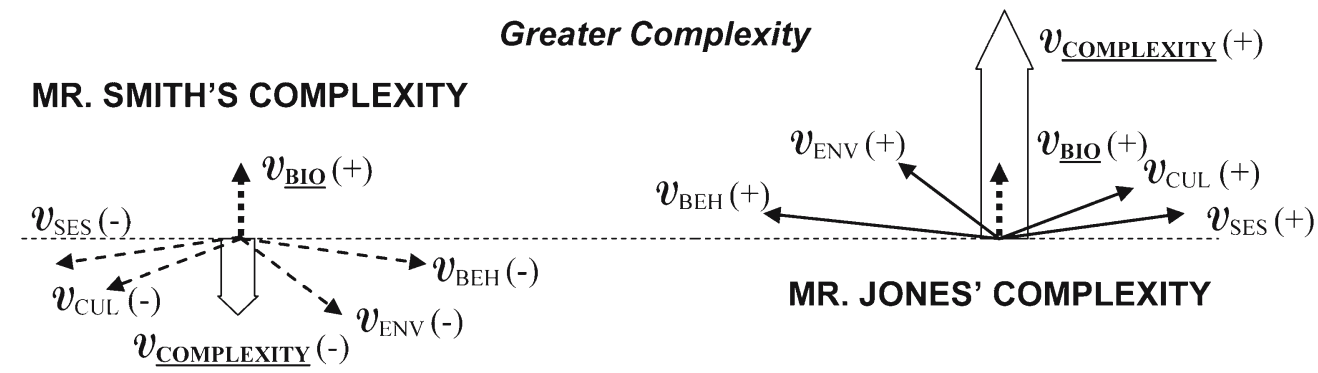

\section{Less Complexity}

Figure 4. Complexity vectors for Mr. Smith and Mr. Jones. The arrows represent vector forces (V). In vector physics, these arrows can be added in space (for more details, see Appendix), and we propose an analogous relationship between the various domains of health determinants. The biological axis is a traditional focus, therefore it is dashed. Greater complexity along the biological axis is toward the top of the diagram, lesser complexity toward the bottom. For both patients, the biological complexity vector $\left(V_{B I O}\right)$ has the same magnitude and direction, but the other vectors differ markedly. The block arrows represent the summary vectors along the biological axis, which for Mr. Smith indicate less overall complexity compared with the biological complexity vector alone (arrow points down). However, for Mr. Jones, the summary vector indicates greater overall complexity compared with the biological complexity vector alone (arrow points up). BIOL biological, SES socioeconomic, CUL cultural, ENV environmental, BEH behavioral 
individuals more prone to treatment-induced harm than others? How does overall risk/benefit assessment change with complexity? What is the incremental benefit of achieving progress toward an ideal risk factor level? What is the overall health effect of multiple interventions implemented simultaneously? Does this differ as the number of medical conditions rises? What is the relative effectiveness of different interventions? Evidence syntheses, such as the CDC's report on the relative cost-effectiveness of hypertension, lipid and glycemic control interventions in older type 2 diabetes patients are helpful but rare. ${ }^{39}$

\section{Guidelines for Complex Patients}

Currently available guidelines tend to focus on single diseases and not on the needs of complex patients, ${ }^{1-3}$ a striking finding on perusal of the over 2,000 available guidelines on the Agency for Healthcare Research and Quality's National Guideline Clearinghouse website. ${ }^{40}$ Notable exceptions include the Veterans Affairs/Department of Defense (VA/DoD) Diabetes Guideline (advising adjusting targets for glycemic control based on life expectancy) and the California Healthcare Foundation/ American Geriatrics Society's (CHF/AGS) diabetes guidelines (1) (which advise prioritizing care among multiple conditions).

In fact, guidelines are almost invariably silent about clinical trade-off decisions. ${ }^{3}$ The CHF/AGS diabetes guidelines are a notable exception in their recommendation to consider more liberal glycemic control targets and greater focus on hypertension control in older diabetic patients. ${ }^{1}$ However, more typical is advice in the Expert Consensus Document on Beta-Adrenergic Receptor Blockers ${ }^{41}$ for patients with chronic obstructive pulmonary disease vis-à-vis beta blockers. Note is made that such patients do benefit, but they should be "carefully monitored" without further guidance on what type of monitoring is recommended, or which specific characteristics identify patients at particular risk.

Trade-off decisions arise along each axis of complexity. Even when sociocultural issues are mentioned in guidelines, specific recommendations for how to address them are uncommon. For example, the Guideline for Family-Centered Care and the Pediatrician's Role recommends "honoring racial, ethnic, cultural, and socioeconomic diversity and its effect on the family's experience and perception of care", but offers no specific advice. ${ }^{40}$ Notable exceptions are the National Health Care for the Homeless Council's seven condition-specific guidelines and one broader guideline for the primary care of homeless individuals. ${ }^{42,43}$

Left to themselves, patients make their own trade-off decisions. Consider diabetes self-care. The American Diabetes Association recommends $2 \mathrm{~h}$ of self-care tasks daily, ${ }^{44}$ unrealistic for many people, especially for a truck driver like Mr. Jones, who may routinely drive $10-11 \mathrm{~h}$ daily. ${ }^{45}$ Communitydwelling diabetic patients reported spending on average $1 \mathrm{~h}$ daily on self-care. ${ }^{46}$ However, diabetic women spent excess time on foot care and too little on exercise, suggesting that medical guidance on trade-off decisions may better optimize health. ${ }^{46}$ Diabetes guidelines offer no recommendations for prioritizing patients' self-care time. ${ }^{44}$

Physicians, too, may make suboptimal trade-off decisions, underutilizing some interventions in complex patients and overusing others. Physicians of older women with chronic stable angina who had $40 \%$ the mammography screening rates of healthy women might have overestimated the influence of angina on life expectancy. ${ }^{47}$ In contrast, up to one third of men over 75 undergo potentially unneeded prostate cancer screening; their life expectancy is limited. ${ }^{48}$ Physicians prioritized glycemic control over blood pressure control in diabetic patients, ${ }^{49}$ despite evidence that aggressive blood pressure control is cost-saving in older patients with type 2 diabetes and tight glycemic control is far less cost-effective. ${ }^{39}$ Physicians may also contribute to lower rates of potentially beneficial services among socioeconomically or culturally complex patients. $^{50,51}$

\section{Quality Measurement for Complex Patients}

Given that evidence suggests suboptimal care, a systematic approach to assessing the quality of care received by complex patients is warranted. In fact, currently unmeasured aspects of care may be especially relevant to complex patients. Efforts to reflect a more global assessment of overall quality of care are hampered by lack of consensus on important methodologic issues, such as how to adjust for patients characteristics or create summary measures. Patients themselves may be queried, but cost and biases inherent in surveying patients are considerable.

Quality measures tend to focus on single diseases without differentiation for complex patients. The VA/DoD, whose Diabetes Guidelines recognize complexity imposed by limited life expectancy, has an opportunity to construct quality measures based on life expectancy, alongside their current age-limited performance measures. The VA has become a widely recognized leader in quality and performance, thus could play an important leadership role in incorporating complexity into performance measures.

Because trade-off decisions are so central to the care of complex patients, incorporating patients' preferences becomes essential for high quality care. For example, Mr. Jones and his physician may liberalize some treatment goals to create a realistic, achievable program of care. Quality metrics do not currently include assessment of patient preferences, although important work in this area has begun and validated scales assess patient opinions about the success of various aspects of the clinical encounter in meeting their personal needs. ${ }^{52,53} \mathrm{We}$ echo others' concerns that the Pay-for-Performance program creates a possible disincentive to elicit patient preferences and the development of an individualized care $\operatorname{plan}^{2-4}$; if physician income is tied to the proportion of patients with $\mathrm{HbAlc}<7 \%$, glycemic control targets may not be the result of shared decision-making.

Patient complexity has been incorporated to some extent into widely implemented reimbursement schemes, offering potential lessons for quality measurement. The Diagnosis Related Group system reimburses more for hospital stays for complicated patients based on the presence of comorbid conditions or complications. Similarly, outpatient visit reimbursement for Medicare patients is tied to the level of visit complexity, based on the number of a defined set of elements addressed, illness severity, and amount of time spent. Note that nonbiological complexity is only indirectly incorporated in the time component of this scheme.

While overcoming many of these challenges requires longterm investment, several possible short-term approaches using available methods are worth noting. Stratifying mea- 
sures on life expectancy, using well-established methods, ${ }^{54-57}$ could include many older patients currently excluded from quality assessment. Peer-based benchmarking is another welldeveloped approach that could be more widely applied, and inherently accounts for complexity. ${ }^{58}$ Peer-based benchmarking compares the performance of an individual to their peer group using sound statistical methods. The lack of evidence on what is realistically achievable is an uncomfortable undercurrent in the quality industry, which often sets benchmarks by expert opinion in the absence of empiric evidence. ${ }^{59}$ The extent to which low performance among even the best performing clinicians is driven by patient complexity is an empirical question worth examining.

\section{IMPLICATIONS FOR PRACTICE, POLICY, AND RESEARCH}

1. Practice implications: Evidence and guidelines. Most pressingly, we need studies that provide data for clinical tradeoff decisions: on the relative effectiveness of interventions within and across diseases, by increments of disease control, and more "real world" effectiveness studies such as the ALLHAT. More evidence synthesis studies that can model time to benefit and relative benefits ${ }^{39}$ are also essential. New evidence and evidence syntheses should be incorporated into guidelines, using the Vector Model of Complexity as a framework. Disease-specific guidelines could consider how complexity along each of the Vector Model's axes might change recommendations, with guidance for trade-off decisions. General guidelines for the care of complex patients with advice on how to assess and address each axis of the Vector Model are also needed.

2. Policy implications: Incorporate complexity into quality measurement and performance profiling. Strategies discussed above that could be implemented in the short term include stratifying quality measures on life expectancy, ${ }^{54-57}$ with new quality measures for individuals with decreased life expectancy, and wider implementation of peer benchmarking as an alternative to arbitrary benchmarks. In the longer term, a more comprehensive assessment of quality of care for complex patients along multiple axes is needed. Specific strategies for how to create metrics to assess the care of complex patients are important areas for future work, for which the Vector Model can serve as a conceptual framework.

3. Research implications: Multidisciplinary teams, standards for clinical trials. The assessment of nonbiological axes, the relative contribution of each axis to overall complexity, and the interrelatedness of each complexity vector require multiple scientific perspectives and methodologies, as emphasized in the current NIH roadmap.

Sociologists, education theorists, complexity scientists, and experts in neural networks are some of the potential team members who might work alongside physicians and biostatisticians to carry out multidisciplinary studies on patient complexity in healthcare. This research should first develop validated methods for quantifying patient complexity along each axis, empirically determining appropriate weighting of the relative contribution of each axis. This may be followed by the development of complexity metrics for quality assessment, and include patient preferences in the metrics. New standards for clinical trials could mandate the reporting of incremental benefits and quality of life outcomes, providing a long-term solution for the knowledge gap that currently hampers evidencebased trade-off decision-making.

\section{CONCLUSION}

Patients may exhibit complexity along any of several axes of the Vector Model of Complexity, which are interrelated in subtle ways. If the multiple forces of complexity are not recognized and addressed, they become barriers to reaching congruence between patient and provider, leading to nonadherence, and diminishing the physician's effectiveness on optimizing health. A rigid, unaccommodating healthcare system can further prevent the achievement of congruence. Achieving congruence requires trade-off decisions, yet there is a distressing paucity of empiric evidence to guide such decisions. Trade-off decisions are largely ignored by current evidence-based guidelines and quality measurement systems. We urgently need to expand the evidence base to inform clinical trade-off decisions, and interdisciplinary teams need to develop methods to measure patient complexity and to integrate such measurement into guidelines and quality assessment. Our Vector Model offers a framework for this future work.

Acknowledgement: We thank Drs. Carol Ashton, Stefan Kertesz, and Nelda Wray, for their helpful comments on an early draft of the manuscript. This paper was presented at the Veterans Affairs Health Services Research and Development State of the Art Conference VIII: Managing Complexity in Chronic Care, September 13-15, 2006 in Arlington, Virginia and at the Academy Health Annual Research Meeting, June 3-5, 2007 in Orlando, Florida. This work was made possible by support from the Veterans Affairs Health Services Research \& Development Research Enhancement Award Program (Kiefe, PI), NIDDK R18DK65001-01AZ (Allison, Safford), and VA HSRAD IIR04-266 (Safford).

Conflict of Interest Disclosure: None disclosed.

Corresponding Author: Monika M. Safford, MD; University of Alabama at Birmingham, 1717 11th Avenue South, MT643, Birmingham, AL 35294-4410, USA (e-mail: msafford@uab.edu).

\section{APPENDIX}

\section{Summing vectors}

Vectors can be summed in space as follows. Consider 3 vectors $\mathrm{V}_{\mathrm{BIOL}}, \mathrm{V}_{\mathrm{CUL}}$, and $\mathrm{V}_{\mathrm{SES}}$ as shown.

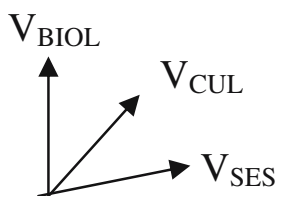


We start with the 2 vectors $\mathrm{V}_{\mathrm{CUL}}$ and $\mathrm{V}_{\mathrm{SES}}$. In order to sum them, we construct a parallelogram and determine its long axis.

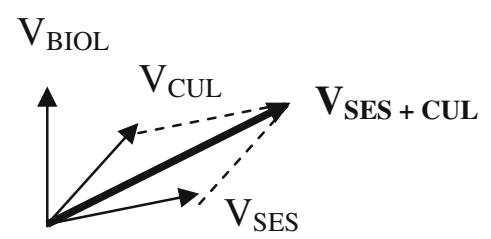

In order to find the sum of all 3 vectors, we construct another parallelogram using $\mathrm{V}_{\mathrm{SES}}+\mathrm{CUL}$ and $\mathrm{V}_{\mathrm{BIOL}}$.

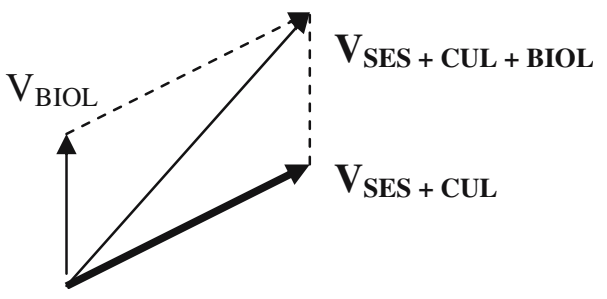

\section{RELATIVE SUMMATION OF VECTORS TO OBTAIN THE TOTAL COMPLEXITY ALONG ANY AXIS}

For a given health care practitioner, the focus of care is along primarily one axis. For physicians, that is usually the biological axis. The question for the physician is: how does complexity along the other axes influence the patient's biological management at this encounter?

This question can be answered by determining the component of a given vector along the biological axis. In the example above, let us first determine the component of the cultural vector that contributes complexity to the biological axis.

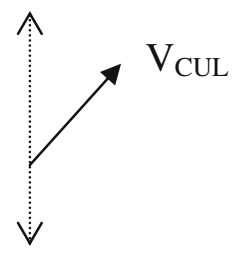

BIOL

The relative contribution of $\mathrm{V}_{\mathrm{CUL}}$ along the biological axis is evaluated by dropping a perpendicular line from the end of $\mathrm{V}_{\mathrm{CUL}}$ onto the biological axis. This component of $\mathrm{V}_{\mathrm{CUL}}$ can be thought of as $\left(\mathrm{V}_{\mathrm{CUL}}\right)$ BIOL

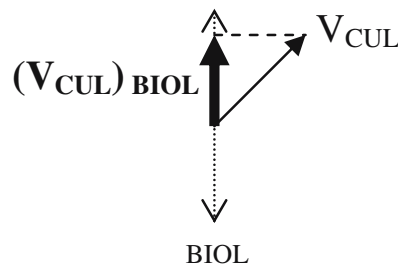

To find the total effect on complexity along the biological axis, the $\left(\mathrm{V}_{\mathrm{CUL}}\right)_{\mathrm{BIOL}}$ can be added to the biological complexity vector as follows:

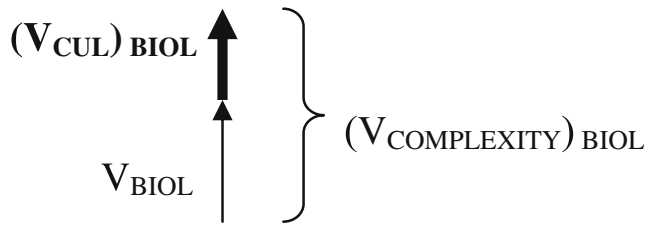

In this example, the total complexity along the biological axis $\left[\left(\mathrm{V}_{\mathrm{COMPLEXITY}}\right)_{\mathrm{BIOL}}=\mathrm{V}_{\mathrm{BIOL}}+\left(\mathrm{V}_{\mathrm{CUL}}\right)_{\mathrm{BIOL}}\right]$ is made greater by cultural complexity. Cultural concordance and high levels of trust can create a vector along the cultural axis that diminishes biological complexity. The direction of the cultural vector will be negative, and the summary force of complexity along the biological axis will be diminished compared to the biological vector alone.<smiles>CC1CCC(C)C1C(C)(C)C</smiles>

BIOL

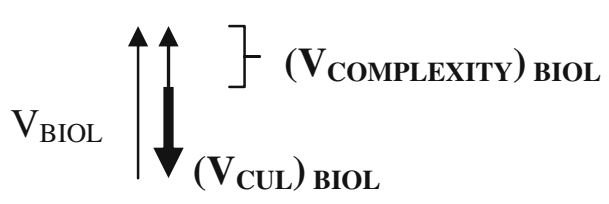

This same procedure can be repeated for each of the vectors present along any axis to arrive at a complexity vector that reflects the effect on the biological complexity vector of all present complexity vectors.

Note that this principle of summation can be applied to any axis. A behavioral scientist will likely be interested in total complexity along the behavioral axis, an anthropologist in the total complexity along the cultural axis, etc.

\section{REFERENCES}

1. Brown AF, Mangione CM, Saliba D, Sarkisian CA. California healthcare foundation/American geriatrics society panel on improving care for elders with diabetes. Guidelines for improving the care of the older person with diabetes mellitus. J Am Geriatr Soc. 2003;May 51(5 Suppl Guidelines):S265-80.

2. Durso sc. Using clinical guidelines designed for older adults with diabetes mellitus and complex health status. JAMA. 2006;295:193540. Apr 26.

3. Tinetti ME, Bogardus ST, Agostini JV. Potential pitfalls of diseasespecific guidelines for patients with multiple conditions. N Engl J Med. 2004;351:2870-4.

4. Boyd CM, Darer J, Boult C, Fried LP, Boult L, Wu AW. Clinical practice guidelines and quality of care for older patients with multiple comorbid diseases: implications for pay for performance. JAMA. 2005;294:716-24. Aug 10 
5. Anderson G, Horvath J. Chronic conditions: Making the case for ongoing care. New Jersey, Robert Wood Johnson Foundation's Partnership for Solutions; 2002.

6. Tarlov AR. Public policy frameworks for improving population health Ann N Y Acad Sci. 1999;896:281-93.

7. Lynch J, Smith GD. A life course approach to chronic disease epidemiology. Annu Rev Public Health. 2005;26:1-35.

8. Astin JA, Forys K. Psychosocial determinants of health and illness: integrating mind, body, and spirit. Adv Mind Body Med. 2004;20:14-21. Winter.

9. Mookadam F, Arthur HM. Social support and its relationship to morbidity and mortality after acute myocardial infarction: systematic overview. Arch Intern Med. 2004;164:1514-8. Jul 26.

10. Starfield B. Measurement of outcome: a proposed scheme. Milbank Mem Fund $\mathrm{Q}$ Health Soc. 1974;52:39-50. Winter.

11. Krieger $\mathbf{N}$. The ostrich, the albatross, and public health: an ecosocial perspective-or why an explicit focus on health consequences of discrimination and deprivation is vital for good science and public health practice. Public Health Rep. 2001;116:419-23. Sep-Oct.

12. Mechanic D. Social psychologic factors affecting the presentation of bodily complaints. N Engl J Med. 1972;286:1132-7.

13. Kleinman AM, Eisenberg L, Good B. Culture, illness and care. Clinical lessons from anthropological and cross-cultural research. Ann Intern Med. 1978;88:251-8.

14. Starfield B, Wray C, Hess $\mathbf{K}$, Gross R, Birk PS, D'Lugoff BC. The influence of patient-practitioner agreement on outcome of care. Am J Public Health. 1981;71:127-32.

15. Mechanic D. Health and illness behavior and patient-practitioner relationships. Soc Sci Med. 1992;12:1345-50.

16. Helman CG. Communication in primary care: the role of patient and practitioner explanatory models. Soc Sci Med. 1985;20:923-31.

17. Frank E, Kupfer DJ, Siegel LR. Alliance not compliance: a philosophy of outpatient care. J Clin Psychiatry. 1995;56(suppl):11-7.

18. Krousel-Wood M, Hyre A, Muntner P, Morisky D. Methods to improve medication adherence in patients with hypertension: current status and future directions. Curr Opin Cardiol. 2005;20:296-300.

19. Pena Dolhun E, Munoz C, Grumbach K. Cross-cultural education in US medical schools: development of an assessment tool. Acad Med. 2003;78:615-22.

20. ALLHAT Officers and Coordinators for the ALLHAT Collaborative Research Group. The Antihypertensive and Lipid-Lowering Treatment to Prevent Heart Attack Trial. Major outcomes in moderately hypercholesterolemic, hypertensive patients randomized to pravastatin vs usual care: The Antihypertensive and Lipid-Lowering Treatment to Prevent Heart Attack Trial (ALLHAT-LLT). JAMA. 2002;288:2998-3007.

21. Savage PJ, Pressel SL, Curb JD, et al. Influence of long-term, low-dose, diuretic-based, antihypertensive therapy on glucose, lipid, uric acid, and potassium levels in older men and women with isolated systolic hypertension: The Systolic Hypertension in the Elderly Program. SHEP Cooperative Research Group. Arch Intern Med. 1998;158:741-51.

22. Costantini O, Huck K, Carlson MD, et al. Impact of a guideline-based disease management team on outcomes of hospitalized patients with congestive heart failure. Arch Intern Med. 2001;161:177-82

23. Pitt B, Zannad F, Remme WJ, et al. The effect of spironolactone on morbidity and mortality in patients with severe heart failure. Randomized Aldactone Evaluation Study Investigators. N Engl J Med. 1999;341:709-17.

24. UKPDS. Tight blood pressure control and risk of macrovascular and microvascular complications in type 2 diabetes: UKPDS 38. UK Prospective Diabetes Study Group. BMJ. 1998;317:703-13.

25. Bauer MS, McBride L, Williford Wo, et al. Cooperative Studies Program 430 Study Team. Collaborative care for bipolar disorder: Part II. Impact on clinical outcome, function, and costs. Psychiatr Serv. 2006;57(7):937-45.

26. Cokkinides V, Bandi P, Ward E, Jemal A, Thun M. Progress and opportunities in tobacco control. CA Cancer J Clin. 2006;56:135-42.

27. National Heart, Lung, and Blood Institute. Clinical Guidelines on the Identification, Evaluation, and Treatment of Overweight and Obesity in Adults. Available at http://www.nhlbi.nih.gov/guidelines/obesity/ ob_home.htm. Accessed August 16, 2006.

28. Wright JT Jr, Bakris G, Greene T, et al. African American study of kidney disease and hypertension study group. Effect of blood pressure lowering and antihypertensive drug class on progression of hypertensive kidney disease: results from the AASK trial. JAMA. 2002;288:2421-31.

29. Nguyen WT, Stewart C, Fisher $\mathbf{K}$, et al. Maintenance asthma treatment with fluticasone/salmeterol combination via Diskus: effect on outcomes in inner-city children enrolled in TennCare. Allergy Asthma Proc. 2005;26: 129-34.

30. Cloutier MM, Hall CB, Wakefield DB, Bailit H. Use of asthma guidelines by primary care providers to reduce hospitalizations and emergency department visits in poor, minority, urban children. J Pediatr. 2005; 146:591-7.

31. Atkins MS, Frazier SL, Birman D, et al. School-based mental health services for children living in high poverty urban communities. Adm Policy Ment Health. 2006;33:146-59.

32. Albright CL, Pruitt L, Castro C, Gonzalez A, Woo S, King AC. Modifying physical activity in a multiethnic sample of low-income women: one-year results from the IMPACT (Increasing Motivation for Physical ACTivity) project. Ann Behav Med. 2005;30(3):191-200.

33. Emmons KM, Stoddard AM, Fletcher R, et al. Cancer prevention among working class, multiethnic adults: results of the healthy directions-health centers study. Am J Public Health. 2005;95:1200-5.

34. Wagner EH, Austin BT, Von Korff M. Organizing care for patients with chronic illness. Milbank Mem Fund Q. 1996;74:511-44.

35. Willenbring $\mathbf{M L}$, Olson $\mathbf{D H}$. A randomized trial of integrated outpatient treatment for medically ill alcoholic men. Arch Int Med. 1999;159 (16): 1946-52.

36. Samet JH, Friedmann $\mathbf{P}$, Saitz $\mathbf{R}$. Benefits of linking primary medical care and substance abuse services. Arch Intern Med. 2001;161:85-91.

37. Kertesz SG, Posner MA, O'Connell JJ, et al. Post-Hospital Medical Respite Care and Readmission of Homeless Persons. J Prev Interv Comm. 2007, in press.

38. Clancy DE, Brown SB, Magruder DM, Huang P. Group visits in medically and economically disadvantaged patients with type 2 diabetes and their relationships to clinical outcomes. Top Health Inf Manage 2003;24:8-14.

39. CDC Diabetes Cost-effectiveness Group. Cost-effectiveness of intensive glycemic control, intensified hypertension control, and serum cholesterol level reduction for type 2 diabetes. JAMA. 2002;287(19):2542-51.

40. Agency for Healthcare Research and Guality. National Guideline Clearinghouse. Available at http://www.guideline.gov/resources/ glossary.aspx. Accessed August 16, 2006.

41. Lopez-Sendon J, Swedberg K, McMurray J, et al. Expert consensus document on beta-adrenergic receptor blockers. Eur Heart J. 2004;25 (15): 1341-62. Aug.

42. National Health Care for the Homeless Council. Practice adaptations. Available at http://www.nhchc.org/practiceadaptations.html. Accessed November 10, 2006.

43. Montauk SL. The homeless in America: adapting your practice. Am Fam Physician. 2006;74:1132-8

44. Russell LR, Suh DC, Safford M. Time requirements for diabetes selfmanagement: too much for many? J Fam Pract. 2005;54:52-6.

45. US Department of Labor Bureau of Labor Statistics. Career Guide to Industries: Truck Transportation and Warehousing. Available at http:// www.bls.gov/oco/cg/cgs021.htm\#conditions. Accessed November 10, 2006.

46. Safford MM, Russell LB, Suh DC, Roman S, Pogach L. How much time do patients with diabetes spend on self-care? J Am Board Fam Pract. 2005; 18:262-70.

47. Kiefe CI, Funkhouser E, Fouad MN, May DS. Chronic disease as a barrier to breast and cervical cancer screening. J Gen Intern Med. 1998; 13:357-65.

48. Scales CD Jr, Curtis LH, Norris RD, Schulman KA, Albala DM, Moul JW. Prostate specific antigen testing in men older than 75 yeears in the US. J Urol. 2006;176:511-4.

49. Merz CN, Buse JB, Tuncer D, Twillman GB. Physician attitudes and practices and patient awareness of the cardiovascular complications of diabetes. J Am Coll Cardiol. 2002;40:1877-81.

50. Rao SV, Schulman KA, Curtis LH, Bersh BJ, Jollis JG. Socioeconomic status and outcome following acute myocardial infarciton in elderly patients. Arch Intern Med. 2004;164:1128-33.

51. Schulman KA, Berlin JA, Harless W, et al. The effect of race and sex on physicians' recommendations for cardiac catheterization. N Engl J Med. 1999;340:618-26.

52. Lerman CE, Brody DS, Caputo GC, et al. Patients' perceived involvement in care scale: relationship to attitudes about illness and medical care. J Gen Intern Med. 1990;5:29-33.

53. Glasgow RE, Wagner EH, Schaefer J, Mahoney LD, Reid RJ, Greene SM. Development and validation of the patient assessment of chronic illness care (PACIC). Med Care. 2005;43:436-44. 
54. Charlson ME, Pompei P, Ales KL, Mackenzie CR. A new method of classifying prognostic comorbidity in longitudinal studies: development and validation. J Chronic Dis. 1987;40:373-83.

55. Johns Hopkins Bloomberg School of Public Health. The Johns Hopkins University ACG Case-Mix System. Available at http://www. acg.jhsph.edu. Accessed August 16, 2006.

56. Elixhauser A, Steiner C, Harris DR, Coffey RM. Comorbidity measures for use with administrative data. Med Care. 1998;36:8-27.
57. Clark DO, Von Korff M, Saunders K, Baluch WM, Simon GE. A chronic disease score with empirically derived weights. Med Care. 1995;33:783-95.

58. Kiefe CI, Weissman NW, Allison JJ, Farmer R, Weaver M, Williams OD. Identifying achievable benchmarks of care: concepts and methodology. Int J Qual Health Care. 1998;10:443-7.

59. Canto JG, Kiefe CI. Door-to-needle time in myocardial infarction: is there an ideal benchmark? Am Heart J. 2005;150:365-7. 